Shusheng Fu, Department of Mathematics, Fuzhou University, Fuzhou Fujian, China

\title{
ON SOME THEOREMS IN FRACTIONAL CALCULUS FOR SINGULAR FUNCTIONS
}

\begin{abstract}
In this paper we show that there are close relationships amongst Cantor bar totality, non-integer integral and Hausdorff integral. If a singular function $f$ has zero Lipschitz $(1-\nu)$-numbers on a Cantor set $C$ with $H$-dim $C=1-\nu, 0<\nu<1$, then the $1-\nu$ order fractional derivative of $f$ exists almost everywhere on $[0, T]$. Moreover, under strong assumptions on the function $f$ the $1-\nu$ order derivative of $f$ exists everywhere. Consequently, the $\nu$ order fractional integral of $f$ equals $f(0) t^{\nu}$. Using the Concept of th Hausdorff derivative we prove that if a singular function has a Hausdorff derivative on $C$, then the fractional derivative of $f$ exists almost everywhere on $[0, T]$. Finally, under some assumptions on $f$ and $C$, we establish
\end{abstract}

$$
\begin{aligned}
\left(D^{-\nu} f\right)(t) & =\int_{0}^{t} \int_{0}^{u} v^{\nu-1} f_{H}^{\prime}(u-v) d v^{1-\nu} d u \\
& =\int_{0}^{t} u^{1-\nu} J_{\nu}\left(v^{\nu-1} f_{H}^{\prime}(u-v)\right)(u) d u
\end{aligned}
$$

This identity is similar to the identity

$$
\left(D^{-\nu} f\right)(t)=\frac{1}{B_{\nu}} t^{\nu}\left(J_{\nu} f\right)(t)
$$

which professor R. R. Nigmatullin claimed.

Key Words: Fractional integral, Fractional derivative, Hausdorff integral, Hausdorff derivative, Lipschitz number, singular function, Cantor type set

Mathematical Reviews subject classification: Primary: 26A33. Secondary: 26A39, $28 \mathrm{~A} 75$

Received by the editors May 10, 1995

* Supported by the Chinese National Science Foundation No. 19671018 


\section{Introduction}

In his paper R. R. Nigmatullin [7] pointed out that there is a relationship between Cantor bar totality and non-integer integral. He claimed that

$$
\left(D^{-\nu} f\right)(t)=\frac{1}{B_{\nu}} t^{\nu}\left(J_{\nu} f\right)(t) B_{\nu} \text { constant }
$$

where

$$
\begin{aligned}
\left(D^{-\nu} f\right)(t) & =\frac{1}{\Gamma(\nu)} \int_{0}^{t}(t-u)^{\nu-1} f(u) d u \\
\left(J_{\nu} f\right)(t) & =\lim _{n \rightarrow \infty} \frac{1}{(2 \xi)^{n} t} \int_{0}^{t} d \tau \sum_{m=1}^{2^{n}} \eta\left(t_{2 m-1}^{(n)}<\tau<t_{2 m}^{(n)}\right) f(\tau),
\end{aligned}
$$

see [7, p. 248]. Some mathematicians doubt identity (1). R. S. Rutman [8] and S. Kempfle [4] showed that in fact (1) is not true. In my previous work [2] I have shown that the whole convolutional integral $\left(J_{\nu} f\right)(t)$ is equivalent to the Hausdorff integral. In this paper we show that there are close relationships amongst Cantor bar totality, non-integer integral and Hausdorff integral. Moreover, we will give an identity which is similar to the identity which R. R. Nigmatallin claimed. The identity is

$$
\left(D^{-\nu} f\right)(t)=\int_{0}^{t} u^{1-\nu} J_{\nu}\left(v^{\nu-1} f_{H}^{\prime}(u-v)\right)(u) d u .
$$

B. B. Mandelbrot states "any nondecreasing function can be written as the sum of a singular function, of a function made of discrete Jumps, and a differentiable function. The last two components are classical in mathematics and of wide use in physics. On the other hand, the singular component is widely regarded in physics as pathological and totally devoid of uses. A principal theme of this Essay is that this last opinion is totally devoid of merit". Let us consider the evolution of some physical systems with losses. In the process there is no loss almost all the time, but there are losses in exceptional time. (The exceptional time subset has uncountable elements but its Lebesgue measure is zero.) Therefore, the functions which describe this phenomenon remain constant in most time and vary in exceptional time. The Cantor type functions (a kind of singular functions) possess this property; so they are suitable for studying the phenomenon mentioned above.

We begin with a theorem about the existence of the fractional derivatives. If a singular function $f$ has zero Lipschitz $(1-\nu)$-numbers on a Cantor set $C$ with $H$-dim $C=1-\nu$, then the $1-\nu$ order fractional derivative of $f$ exist 
almost everywhere on $[0, T]$. This answers, in one way, an open problem posed by C. Tricot [10], "Are there any relationships between the Lipschitz $\eta$-number and $\eta$ order fractional derivative of function $f$ ?" Other answers to Tricot's question were given by Hardy and Littlewood [3]. Moreover, under strong assumptions on the function $f$ the $\nu$ order derivative of $f$ exist everywhere on $[0, T]$. By elementary Calculus the $1-\nu$ order fractional integral of $f$ equals $f(0) t^{\nu}$. That is, if a Cantor type function $f$ has bounded Lipschitz $(1-\nu+\varepsilon)$ numbers on $C$, then the $\nu$ order integral of $f$ is equal to the power function $f(0) t^{\nu}$. Using the concept of Hausdorff derivative we prove that if a Cantor type function $f$ has a Hausdorff derivative on $C$, then the sequence derivative of the Riemann-Liouville fractional integral of order $\nu$ of the function $f$ exist almost everywhere on $[0, T]$. Finally, under additional conditions on $f$ and $C$, we establish the identity

$$
\left(D^{-\nu} f\right)(t)=\int_{0}^{t} u^{1-\nu} J_{\nu}\left(v^{\nu-1} f_{H}^{\prime}(u-v)\right)(u) d u
$$

\section{Notations and Terminology}

The Cantor dust of dimension $\nu$ is constructed in the usual way. Let $[0, T]$ be an interval and let $0<\xi<\frac{1}{2}$. A partition of $[0, T]$ is given by

$$
a_{1}^{1}=0<b_{1}^{1}=\xi T<a_{1}^{2}=(1-\xi) T<b_{1}^{2}=T,
$$

write $U_{1}=U_{1}^{1} \cup U_{1}^{2}$, where $U_{1}^{1}=\left[a_{1}^{1}, b_{1}^{1}\right], U_{1}^{2}=\left[a_{1}^{2}, b_{1}^{2}\right]$. A partition of $U_{1}^{j}$, $j=1,2$, is given by

$$
a_{2}^{1}=a_{1}^{1}<b_{2}^{1}<a_{2}^{2}<b_{2}^{2}=b_{1}^{1}<a_{2}^{3}=a_{1}^{2}<b_{2}^{3}<a_{2}^{4}<b_{2}^{4}=b_{1}^{2},
$$

where

$$
b_{2}^{1}=a_{1}^{1}+\xi^{2} T, \quad a_{2}^{2}=b_{1}^{1}-\xi^{2} T, \quad b_{2}^{3}=a_{1}^{2}+\xi^{2} T, \quad a_{2}^{2}=b_{1}^{2}-\xi^{2} T .
$$

Continue this process to obtain a sequence of intervals $\left\{U_{n}^{i}\right\}_{i=1}^{2^{n}}, n=1,2, \ldots$. Let

$$
U_{n}=\bigcup_{i=1}^{2^{n}} U_{n}^{i}, \text { and } C=\bigcap_{n=1}^{\infty} U_{n} .
$$

We call the closed set $C$ a Cantor dust. It is well known that $m C=0$, $\nu=H$-dim $C=\frac{\log 2}{\log \frac{1}{\xi}}$ and $0<H^{\nu}(C)<\infty$, where $H$-dim denotes Hausdorff dimension and $H^{\nu}$ Hausdorff measure. The points $a_{n}^{i}$ or $b_{n}^{i}, i=1,2, \ldots, 2^{n}$, 
$n=1,2, \ldots$ are end points of $C$; The points $t \in C \backslash \cup\left\{a_{n}^{i}, b_{n}^{i}\right\}$ are interior points of $C$ in the sense of related topology. We call a continuous function $f$ a Cantor type function on $C$ if $f$ is constant on each open interval of $[0, T] \backslash C$. Throughout this paper we will consider Cantor type functions.

The discussion will be related to the concepts of fractional calculus, Hausdorff calculus and others.

\subsection{Fractional Integral and Fractional Derivative}

Let $\nu$ be a real positive number and $f$ be a continuous function. The expression

$$
\left(D^{-\nu} f\right)(t)=\frac{1}{\Gamma(\nu)} \int_{0}^{t} u^{\nu-1} f(t-u) d u
$$

is the Riemann-Liouville fractional integral of order $\nu$ of the function $f$ [10]. The $\nu$ order derivative of the function $f$ is defined as

$$
\left(D^{\nu} f\right)(t)=\frac{d}{d t} \frac{1}{\Gamma(1-\nu)} \int_{0}^{t} u^{-\nu} f(t-u) d u
$$

\subsection{Hausdorff Integral and Hausdorff Derivative}

For any singular function $f$ on $C$ [2], we define the net extreme Hausdorff derivative as

$$
\begin{aligned}
& \overline{N-H D} F(t)=\varlimsup_{n \rightarrow \infty} \frac{f\left(b_{n}^{i_{t}}\right)-f\left(a_{n}^{i_{t}}\right)}{H^{\nu}\left(C \bigcap\left[a_{n}^{i_{t}}, b_{n}^{i_{t}}\right)\right.}, \\
& \underline{N-H D F(t)}=\lim _{n \rightarrow \infty} \frac{f\left(b_{n}^{i_{t}}\right)-f\left(a_{n}^{i_{t}}\right)}{H^{\nu}\left(C \bigcap\left[a_{n}^{i_{t}}, b_{n}^{i_{t}}\right)\right.},
\end{aligned} \quad t \in\left(a_{n}^{i_{t}}, b_{n}^{i_{t}}\right), n=1,2, \ldots
$$

If $\overline{N-H D} f(t)=N-H D f(t)$, we say that the function $f(t)$ is net Hausdorff differentiable at $t$; this limit is termed the net Hausdorff derivative of $f(t)$ at $t$ and is denoted by $f_{H}^{\prime}(t)$.

A real valued function $f$ on $C$ with $H$-dim $C=S$ is said to be Hausdorff integrable on $[a, b]$, if there exists $r$, such that for every $\varepsilon>0$ there is a gauge function $\delta(t)>0$ on $C$, such that for any net $\delta$-fine division $D=\{([u, v], t)\}$ of $C$, we have

$$
\left|(D) \sum f(t) H^{\nu}(C \cap[u, v])-r\right|<\epsilon
$$

As usual, we write $\int_{a}^{b} f(t) d t^{s}=r$. 


\subsection{Sequence Derivative}

Let $\left\{h_{n}\right\}_{n=1}^{\infty}$ be a sequence of positive numbers with $h_{n} \downarrow 0$, let $f:[0, T] \rightarrow \mathbb{R}$ and let $t \in(0, T)$. The function $f$ has a sequence derivative with $\left\{h_{n}\right\}_{n=1}^{\infty}$ at $t$ if both the limits

$$
\lim _{n \rightarrow \infty} \frac{f\left(t-h_{n}\right)-f(t)}{-h_{n}} \text { and } \lim _{n \rightarrow \infty} \frac{f\left(t+h_{n}\right)-f(t)}{h_{n}}
$$

exist and are equal. We will use $(q) \frac{d f}{d t}$ to denote the sequence derivative of $f$ at $t$.

\subsection{Lipschitz Number}

The derivative of the form

$$
L^{s}(f, t)=\lim _{\substack{y, z \rightarrow x \\ y<x<z}} \frac{f(z)-f(y)}{(z-y)^{s}}
$$

following Besicovich [9, p. 1] we call the Lipschitz s-number.

\subsection{Self-Similar Property}

For each $t \in C$, there exists a sequence of intervals $\left\{\left[a_{n}^{i_{t_{n}}}, b_{n}^{i_{t_{n}}}\right]\right\}$ such that $t \in\left[a_{n}^{i_{t_{n}}}, b_{n}^{i_{t_{n}}}\right]$. A Cantor type function $f$ has the self-similar property if there exists a constant $\beta$ such that

$$
\lim _{n \rightarrow \infty} \frac{\int_{a_{n}^{i_{n}}}^{b_{n}^{i_{t_{n}}}}\left[f(u)-f\left(a_{n}^{i_{t_{n}}}\right) d u\right.}{\int_{a_{n}}^{b_{n}^{i_{n}}} f_{H}^{\prime}(u) d u^{s}}=\beta
$$

where $t \in\left[a_{n}^{i_{t_{n}}}, b_{n}^{i_{t_{n}}}\right], t \in C ; \int_{a_{n}^{i_{n}}}^{b_{n}^{i_{t_{n}}}} f_{H}^{\prime}(u) d u^{s} \neq 0$ for each $n$ and $H$-dim $C=S$.

For example the Cantor ternary function has the self-similar property.

\subsection{Notations from Henstock Integration Theory}

Let $I$ be the set of all closed intervals $[u, v]$ in $[a, b]$ having a nonempty interior. An element $([u, v], t) \in I \times[a, b]$ is called an interval-point pair. We write $D=\{[u, v], t\}$ where $([u, v], t)$ denotes a typical interval-point pair in $D$. Then $D$ is said to be a partition of $[a, b](C$ resp. $)$ if $\{[u, v] ;([u, v], t) \in$ $D\}$ is a finite collection of nonoverlapping subintervals of $[a, b]$ and $[a, b]=$ 
$\cup_{([\alpha, \beta], t) \in D}[\alpha, \beta]\left(C=\cup_{([\alpha, \beta], t) \in D}[\alpha, \beta] \cap C\right)$. Let $\delta(t)$ be a positive function on $[a, b](E)$. The partition $D$ is called a $\delta$-fine division of $[a, b](E)$ if $([\alpha, \beta], t) \in D$, then $\beta-\alpha \leq \delta(t)$.

\section{$3 \quad$ Main Result}

We assume for simplicity and without loss of generality that in the following, the Cantor type functions $f$ are nondecreasing.

Theorem 1 Let $f$ be a Cantor type function on $C$ with $H$-dim $C=1-\nu$, $0<\nu<1$. Suppose that the Lipschitz $(1-\nu)$-numbers $L^{1-\nu}(f, t)=0$ uniformly on $C$. Then

$$
D^{1-\nu} f(t)=\frac{f(0)}{\Gamma(\nu)} t^{\nu-1}
$$

for $t \in[0, T) \backslash C$. That is the $1-\nu$ order derivative of the function $f$ exists almost everywhere.

Proof. We can write

$$
\begin{aligned}
\left(D^{-\nu} f(t+h)-D^{-\nu} f(t)=\right. & \frac{1}{\Gamma(\nu)} \int_{0}^{t} u^{\nu-1}[f(t+h-u)-f(t-u)] d u \\
& +\frac{1}{\Gamma(\nu)} \int_{t}^{t+h} u^{\nu-1} f(t+h-u) d u \\
= & I_{1}(t, h)+I_{2}(t, h)
\end{aligned}
$$

where $h>0$. Clearly

$$
\frac{I_{2}(t, h)}{h}=\frac{(t+\theta h)^{\nu-1}}{\Gamma(\nu)} f(\overline{1-\theta} h) \rightarrow \frac{f(0)}{\Gamma(\nu)} t^{\nu-1}
$$

for $h \rightarrow 0$.

We will show that $\frac{I_{1}(t, h)}{h}=o(1)(h \rightarrow 0)$ for $t \in[0, T] \backslash C$. Let $n_{t}$ be an index for which $t \in\left(b_{n_{t}}^{i_{t}}, a_{n_{t}}^{i_{t}+1}\right)$. Let $\varepsilon>0$. Under the assumption on the Lipschitz numbers of $f$, there is a $\delta_{1}>0$ such that

$$
\frac{\left|f\left(u+P_{1}\right)-f\left(u-P_{2}\right)\right|}{\left(P_{1}+P_{2}\right)^{1-\nu}}<\varepsilon_{1}
$$

where $u \in[0, T], \varepsilon_{1}=\frac{\left(\frac{t-b_{n_{t}}^{i_{t}}}{2}\right)^{1-\nu}}{b T^{1-\nu}} \varepsilon, P_{1} \geq 0, P_{2} \geq 0$ and $0<P_{1}+P_{2} \leq \delta_{1}$. 
Let $\delta=\min \left(\delta_{1}, \frac{t-b_{n_{t}}^{i_{t}}}{2}\right)$. For any $h, 0<h<\delta$, choose an index $n$ such that $h_{n} \leq h \leq h_{n-1}$ and $h_{n}=\xi^{n} T$. Then the integral $I_{1}^{\prime}(t, h)\left(I_{1}^{\prime}(t, h)=\right.$ $\left.\frac{1}{\Gamma(\nu)} I_{1}(t, h)\right)$ may be expressed as

$$
\begin{aligned}
\int_{0}^{t-b_{n_{t}}^{i_{t}}}+ & \int_{t-b_{n_{t}}^{i_{t}}}^{t-b_{n_{t}}^{i_{t}}+h}+\cdots+\int_{t-b_{n_{t}}^{i_{t}}+m h}^{t} u^{\nu-1}[f(t+h-u)-f(t-u)] d u \\
= & \int_{I_{1}}+\int_{I_{2}}+\cdots+\int_{I_{m+2}} u^{\nu-1}[f(t+h-u)-f(t-u)] d u \\
= & \sum_{I_{j} \in D_{1}} \int_{I_{j}} u^{\nu-1}[f(t+h-u)-f(t-u)] d u \\
& +\sum_{I_{j} \in D_{2}} \int_{I_{j}} u^{\nu-1}[f(t+h-u)-f(t-u)] d u
\end{aligned}
$$

where $D_{1}$ denotes the collection of all intervals for which the functions $f(t-u)$ and $f(t+h-u)$ are equal for $u \in I_{j}$; and $D_{2}$ the collection of the other terms of $\left\{I_{j}\right\}_{j=1}^{m+2}$. Clearly

$$
\sum_{I_{j} \in D_{1}} \int_{I_{j}} u^{\nu-1}[f(t+h-u)-f(t-u)] d u=0 .
$$

Observe that the number of the terms of $D_{2}$ is less than $3 \times 2^{n}$. Therefore

$$
\left|\sum_{I_{j} \in D_{2}} \int_{I_{j}} u^{\nu-1}[f(t+h-u)-f(t-u)] d u\right| \leq 3 \times 2^{n} \frac{\varepsilon_{1} h^{1-\nu}}{\left(\frac{x-b_{n_{t}}^{i_{t}}}{2}\right)^{1-\nu}} h .
$$

By our choice of the index $n$,

$$
2^{n} h^{1-\nu} \leq 2^{n} h_{n-1}^{1-\nu} \leq 2 \times 2^{n-1} \xi^{(n-1) \frac{\log 2}{\log \frac{1}{\xi}}} T^{1-\nu} \leq 2 T^{1-\nu} .
$$

Hence we have

$$
\left|\frac{\sum_{I_{j} \in D_{2}} \int_{I_{j}} u^{\nu-1}[f(t+h-u)-f(t-u)] d u}{h}\right| \leq \varepsilon_{1} \frac{6 T^{1-\nu}}{\left(\frac{x-b_{n_{t}}^{i_{t}}}{2}\right)^{1-\nu}}=\varepsilon
$$

for $0<h \leq \delta$ as required. The case for $h<0$ is treated in a similar way.

Theorem 2 Let $f$ be a Cantor type function on $C$ with $H$-dim $C=1-\nu$. Suppose that the Lipschitz $r$-numbers $L^{r}(f, t) \leq A 1-\nu<r$ uniformly on $C$. Then

$$
\left(D^{-\nu} f\right)(t)=f(0) t^{\nu}
$$


Proof. Under the assumption on the Lipschitz numbers of $f$, we have

$$
L^{1-\nu}(f, t)=0
$$

uniformly on $C$. By Theorem 1

$$
\left(D^{1-\nu} f\right)(t)=\frac{f(0)}{\Gamma(\nu)} t^{\nu-1}
$$

for $t \in[0, T] \backslash C$. We will show that $D^{1-\nu} f(t)=\frac{f(0)}{\Gamma(\nu)} t^{\nu-1}$ for $t \in C$. Let $r=1-\nu+\eta, \eta>0$, and let $t_{0}=t-\frac{T^{\frac{\eta}{2(1-\nu)}}}{2}$. We may write

$$
\begin{aligned}
D^{-\nu} f(t+h)- & D^{-\nu} f(t) \\
= & \frac{1}{\Gamma(\nu)} \int_{0}^{\frac{T^{\frac{\eta}{2(1-\nu)}}}{2}}+\int_{\frac{T^{2}}{2(1-\nu)}}^{t} u^{\nu-1}[f(t+h-u)-f(t-u)] d u \\
& +\frac{1}{\Gamma(\nu)} \int_{t}^{t+h} u^{\nu-1} f(t+h-u) d u \\
= & I_{1}(t, h)+I_{2}(t, h)+I_{3}(t, h) .
\end{aligned}
$$

Arguing as in the proof of Theorem 1 shows that

$$
\begin{aligned}
& \lim _{h \rightarrow 0} \frac{I_{2}(t, h)}{h}=0, \\
& \lim _{h \rightarrow 0} \frac{I_{3}(t, h)}{h}=\frac{f(0)}{\Gamma(\nu)} t^{\nu-1} .
\end{aligned}
$$

We decompose $I_{1}(t, h)$ into two groups as

$$
I_{1}(t, h)=I_{1.1}(t, h)+I_{1.2}(t, h)
$$

where $I_{1.1}(t, h)$ is the sum of all integrals $\int_{I_{j}} u^{\nu-1}[f(t+h-u)-f(t-u)] d u$ for which the functions $f(t-u)$ and $f(t+h-u)$ are equal for $u \in I_{j}$, and $I_{1.2}(t, h)$ the sum of the other integrals. Consequently, $I_{1,1}(t, h)=0$. We will show that $\lim _{h \rightarrow 0} \frac{I_{1.2}(t, h)}{h}=0$.

By the assumption on the Lipschitz numbers of $f$, there is a $\delta>0$ such that

$$
\frac{\left|f\left(t+P_{1}\right)-f\left(t-P_{2}\right)\right|}{\left(P_{1}+P_{2}\right)^{r}} \leq A
$$

$P_{1} \geq 0, P_{2} \geq 0,0<P_{1}+P_{2} \leq \delta$ and $t \in C$, then $t \in[0, T]$. For any $h>0$, choose an index $n$ such that $h_{n} \leq h<h_{n-1}$. By the symmetric distribution of 
$\left\{\left[a_{n}^{i}, b_{n}^{i}\right]_{i=1}^{2^{n}}\right\}_{n=1,2, \ldots}$ the number of the terms of $I_{1.2}(t, h)$ is less than $3\left[2^{n \frac{\eta}{2(1-\nu)}}\right]$. Consequently

$$
\left|I_{1,2}(t, h)\right| \leq 3\left[2^{n \frac{\eta}{2(1-\nu)}}\right] A \frac{h^{r}}{h^{1-\nu}} h .
$$

Therefore

$$
\begin{aligned}
\left|\frac{I_{1,2}(t, h)}{h}\right| & \leq 3 A 2^{n \frac{\eta}{2(1-\nu)}} h^{\eta} \leq 3 A 2^{n \frac{\eta}{2(1-\nu)}} h_{n-1}^{\eta} \\
& \leq 3 A 2^{n \frac{\eta}{2(1-\nu)}} \frac{T^{\frac{\eta}{2}}}{2^{n \frac{\eta}{2(1-\nu)}}} h_{n-1}^{\frac{\eta}{2}} \leq 3 A T^{\frac{\eta}{2}} h_{n-1}^{\frac{\eta}{2}} .
\end{aligned}
$$

The last inequality shows that $\lim _{h \rightarrow 0} \frac{I_{1.2}(t, h)}{h}=0$ as required.

We have shown that $\left(D^{1-\nu} f\right)(t)=\frac{f(0)}{\Gamma(\nu)} t^{\nu-1}$ for each $t \in[0, T]$. By the elementary calculus we get $\left(D^{-\nu} f\right)(t)=f(0) t^{\nu}$.

Theorem 3 Let $f$ be a Cantor type function on $C$ with $H-\operatorname{dim} C=1-\nu$. Suppose that $f_{H}^{\prime}(t)$ exists and is continuous on $C$ and $f$ has the self-similar property. Then

$$
(q) \frac{d D^{-\nu} f}{d t}(t)=\frac{1}{\Gamma(\nu)} \int_{0}^{t} u^{\nu-1} f_{H}^{\prime}(t-u) d u^{1-\nu}+\frac{f(0)}{\Gamma(\nu)} t^{\nu-1}
$$

for $t \in[0, T] \backslash C$.

Proof. We may write

$$
\begin{aligned}
\left(D^{-\nu} f\left(t+h_{n}\right)-D^{-\nu} f(t)\right. & \\
= & \frac{1}{\Gamma(\nu)} \int_{0}^{t} u^{\nu-1}\left[f\left(t+h_{n}-u\right)-f(t-u)\right] d u \\
& +\frac{1}{\Gamma(\nu)} \int_{t}^{t+h_{n}} u^{\nu-1} f\left(t+h_{n}-u\right) d u \\
= & I_{1}\left(t, h_{n}\right)+I_{2}\left(t, h_{n}\right)
\end{aligned}
$$

where $h_{n}>0$. Clearly

$$
\frac{I_{2}\left(t, h_{n}\right)}{h_{n}}=\frac{\left(t+\theta h_{n}\right)^{\nu-1}}{\Gamma(\nu)} f\left(\overline{1-\theta} h_{n}\right) \rightarrow \frac{f(0)}{\Gamma(\nu)} t^{\nu-1}
$$

as $n \rightarrow \infty$. 
We show that

$$
\lim _{n \rightarrow \infty} \frac{I_{1}\left(t, h_{n}\right)}{h_{n}}=\frac{1}{\Gamma(\nu)} \int_{0}^{t} u^{\nu-1} f_{H}^{\prime}(t-u) d u^{1-\nu} \text { for } t \in[0, T] \backslash C .
$$

Let $n_{t}$ be an index for which $t \in\left(b_{n_{t}}^{i_{t}}, a_{n_{t}}^{i_{t}+1}\right)$. Let $\varepsilon>0$. By the uniformly continuity of the function $u^{\nu-1}$ on $\left[t-b_{n_{t}}^{i_{t}}, T\right]$ there is a $\delta_{1}>0$, such that

$$
\left|u^{\nu-1}-v^{\nu-1}\right|<\varepsilon
$$

whenever $u, v \in\left[t-b_{n_{t}}^{i_{t}}, T\right]$ and $|u-v|<\delta_{1}$. By the self-similarity of $f$, there is a positive integer $N_{1}$ such that

$$
\left|\int_{a_{n}^{i}}^{b_{n}^{i}}[f(u)-f(a)] d u-\beta \int_{a_{n}^{i}}^{b_{n}^{i}} f_{H}^{\prime}(u) d u^{1-\nu}\right|<\varepsilon \int_{a_{n}^{i}}^{b_{n}^{i}} f_{H}^{\prime}(u) d u^{1-\nu}
$$

for $n>N_{1}$. By the continuity of $f$ and $f_{H}^{\prime}$ there is a positive integer $N_{2}$ such that

$$
\begin{array}{r}
\left|\frac{1}{h_{n}} \int_{t-b_{n}^{\prime}}^{t} u^{\nu-1}\left[f\left(t+h_{n}-u\right)-f(t-u)\right] d u\right|<\varepsilon \\
\left|\int_{t-b_{n}^{\prime}}^{t-a_{n}^{\prime}} u^{\nu-1}\left[f_{H}^{\prime}(t-u)\right] d u^{1-\nu}\right|<\varepsilon .
\end{array}
$$

Take an integer $N$ such that $h_{N}<\frac{t-b_{n_{t}}^{i_{t}}}{2}, 2 h_{N}<\delta$ and $N>\max \left(N_{1}, N_{2}\right)$. Let $n>N$. We decompose $I_{1}\left(t, h_{n}\right)$ as

$$
\begin{aligned}
& \left(\int_{t-b_{n}^{m}}^{t-b_{n}^{m}+h_{n}}+\int_{t-b_{n}^{m}+h_{n}}^{t-b_{n}^{m}+2 h_{n}}\right)+\left(\int_{t-b_{n}^{m-1}}^{t-b_{n}^{m-1}+h_{n}}+\int_{t-b_{n}^{m-1}+h_{n}}^{t-b_{n}^{m-1}+2 h_{n}}\right)+\cdots \\
+ & \left(\int_{t-b_{n}^{2}}^{t-b_{n}^{2}+h_{n}}+\int_{t-b_{n}^{2}+h_{n}}^{t-b_{n}^{2}+2 h_{n}}\right)+\int_{t-b_{n}^{\prime}}^{t} u^{\nu-1}\left[f\left(t+h_{n}-u\right)-f(t-u)\right] d u
\end{aligned}
$$

where $b_{n_{t}}^{i_{t}}=b_{n}^{m}$.

First we compute

$$
\begin{aligned}
I_{1, j}= & \int_{t-b_{n}^{j}}^{t-b_{n}^{j}+h_{n}} u^{\nu-1}\left[f\left(t+h_{n}-u\right)-f(t-u)\right] d u \\
& +\int_{t-b_{n}^{j}+h_{n}}^{t-b_{n}^{j}+2 h_{n}} u^{\nu-1}\left[f\left(t+h_{n}-u\right)-f(t-u)\right] d u, \quad 2 \leq j \leq m
\end{aligned}
$$




$$
\begin{aligned}
= & \int_{t-b_{n}^{j}}^{t-b_{n}^{j}+h_{n}} u^{\nu-1}\left[f\left(b_{n}^{j}\right)-f(t-u)\right] d u \\
& +\int_{t-b_{n}^{j}+h_{n}}^{t-b_{n}^{j}+2 h_{n}} u^{\nu-1}\left[f\left(t+h_{n}-u\right)-f\left(a_{n}^{j}\right)\right] d u \\
= & \left(t-b_{n}^{j}+\theta_{j} h_{n}\right)^{\nu-1} \int_{t-b_{n}^{j}}^{t-b_{n}^{j}+h_{n}}\left[f\left(b_{n}^{j}\right)-f(u)\right] d u \\
& +\left(t-b_{n}^{j}+\overline{1+\theta_{j}} h_{n}\right)^{\nu-1} \int_{a_{n}^{j}}^{b_{n}^{j}}\left[f(u)-f\left(a_{n}^{j}\right)\right] d u
\end{aligned}
$$

Note that

$$
\int_{a_{n}^{j}}^{b_{n}^{j}}\left[f\left(b_{n}^{j}\right)-f(u)\right] d u+\int_{a_{n}^{j}}^{b_{n}^{j}}\left[f(u)-f\left(a_{n}^{j}\right)\right] d u=\left[f\left(b_{n}^{j}\right)-f\left(a_{n}^{j}\right)\right]\left(b_{n}^{j}-a_{n}^{j}\right) .
$$

We have

$$
\begin{aligned}
I_{1 . j}= & \left(t-b_{n}^{j}+\theta_{j} h_{n}\right)^{\nu-1}\left[f\left(b_{n}^{j}\right)-f\left(a_{n}^{j}\right)\right]\left(b_{n}^{j}-a_{n}^{j}\right) \\
& +\left[\left(t-b_{n}^{j}+\overline{1+\theta_{j}} h_{n}\right)^{\nu-1}-\left(t-b_{n}^{j}+\theta_{j} h_{n}\right)^{\nu-1}\right] \int_{a_{n}^{j}}^{b_{n}^{j}}\left[f(u)-f\left(a_{n}^{j}\right)\right] d u .
\end{aligned}
$$

Using the above identity we show that

$$
\frac{I_{1}\left(t, h_{n}\right)}{h_{n}}-\frac{1}{\Gamma(\nu)} \int_{0}^{t} u^{\nu-1} f_{H}^{\prime}(t-u) d u^{1-\nu}=o(1) .
$$

Because

$$
\int_{0}^{t} u^{\nu-1} f_{H}^{\prime}(t-u) d u^{1-\nu}=\sum_{j=1}^{m} \int_{t-b_{n}^{j}}^{t-a_{n}^{j}} u^{\nu-1} f_{H}^{\prime}(t-u) d u^{1-\nu},
$$

we may write

$$
\frac{I_{1}\left(t, h_{n}\right)}{h_{n}}-\int_{0}^{t} u^{\nu-1} f_{H}^{\prime}(t-u) d u^{1-\nu}
$$

as

$$
\begin{aligned}
& \sum_{j=2}^{m}\left[\left(t-b_{n}^{j}+\theta_{j} h_{n}\right)^{\nu-1} \int_{a_{n}^{j}}^{b_{n}^{j}} f_{H}^{\prime}(u) d u^{1-\nu}-\int_{t-b_{n}^{j}}^{t-a_{n}^{j}} u^{\nu-1} f_{H}^{\prime}(t-u) d u^{1-\nu}\right] \\
& +\sum_{j=2}^{m}\left[\left(t-b_{n}^{j}+\overline{1+\theta_{j}} h_{n}\right)^{\nu-1}-\left(t-b_{n}^{j}+\theta_{j} h_{n}\right)^{\nu-1}\right]
\end{aligned}
$$




$$
\begin{aligned}
& \times\left[\frac{1}{h_{n}} \int_{a_{n}^{j}}^{b_{n}^{j}}\left[f(u)-f\left(a_{n}^{j}\right)\right] d u-\beta \int_{a_{n}^{j}}^{b_{n}^{j}} f_{H}^{\prime}(u) d u^{1-\nu}\right] \\
& +\beta \sum_{j=2}^{m}\left[\left(t-b_{n}^{j}+\overline{1+\theta_{j}} h_{n}\right)^{\nu-1}-\left(t-b_{n}^{j}+\theta_{j} h_{n}\right)^{\nu-1}\right] \int_{a_{n}^{j}}^{b_{n}^{j}} f_{H}^{\prime}(u) d u^{1-\nu} \\
& +\frac{1}{h_{n}} \int_{t-b_{n}^{1}}^{t} u^{\nu-1}\left[f\left(t+h_{n}-u\right)-f(t-u)\right] d u-\int_{t-b_{n}^{1}}^{t-a_{n}^{1}} u^{\nu-1} f_{H}^{\prime}(t-u) d u^{1-\nu} .
\end{aligned}
$$

This expression combining (2), (3), (4), (5) yields

$$
\begin{aligned}
& \left|\frac{I_{1}\left(t, h_{n}\right)}{h_{n}}-\int_{0}^{t} u^{\nu-1} f_{H}^{\prime}(t-u) d u^{1-\nu}\right| \\
\leq & \sum_{j=2}^{m} \int_{a_{n}^{j}}^{b_{n}^{j}}\left|\left(t-b_{n}^{j}+\theta_{j} h_{n}\right)^{\nu-1}-(t-u)^{\nu-1}\right| f_{H}^{\prime}(u) d u^{1-\nu} \\
& +\varepsilon \sum_{j=2}^{m}\left|\left(t-b_{n}^{j}+\overline{1+\theta_{j}} h_{n}\right)^{\nu-1}-\left(t-b_{n}^{j}+\theta_{j} h_{n}\right)^{\nu-1}\right| \int_{a_{n}^{j}}^{b_{n}^{j}} f_{H}^{\prime}(u) d u \\
& +\beta \sum_{j=2}^{m}\left|\left(t-b_{n}^{j}+\overline{1+\theta_{j}} h_{n}\right)^{\nu-1}-\left(t-b_{n}^{j}+\theta_{j} h_{n}\right)^{\nu-1}\right| \int_{a_{n}^{j}}^{b_{n}^{j}} f_{H}^{\prime}(u) d u \\
& +\frac{1}{h_{n}} \int_{t-b_{n}^{1}}^{t} u^{\nu-1}\left|f\left(t+h_{n}-u\right)-f(t-u)\right| d u \\
& +\int_{t-b_{n}^{1}}^{t-a_{n}^{1}} u^{\nu-1} f_{H}^{\prime}(t-u) d u^{1-\nu} \\
\leq & \varepsilon \int_{0}^{t} f_{H}^{\prime}(u) d u^{1-\nu}+\varepsilon^{2} \int_{0}^{t} f_{H}^{\prime}(u) d u^{1-\nu}+\beta \varepsilon \int_{0}^{t} f_{H}^{\prime}(u) d u^{1-\nu}+2 \varepsilon \\
=\varepsilon & {\left[(1+\varepsilon+\beta) \int_{0}^{t} f_{H}^{\prime}(u) d u^{1-\nu}+2\right] }
\end{aligned}
$$

as required.

Remark 1 The Hausdorff integral $\int_{0}^{t} u^{\nu-1} f_{H}^{\prime}(t-u) d u^{1-\nu}$ exists for $t \in[0, T] \backslash C$, since the integrand function $u^{\nu-1} f_{H}^{\prime}(t-u)$ is a continuous function.

Remark 2 Let $G(t)=\int_{0}^{t} u^{\nu-1} f_{H}^{\prime}(t-u) d u^{1-\nu}$. Then $G$ is defined on each interval $\left(b_{n}^{i}, a_{n}^{i+1}\right], i=1,2, \ldots, 2^{n}, n=1,2, \ldots$ Since $\left|\int_{0}^{t} u^{\nu-1} f_{H}^{\prime}(t-u) d u^{1-\nu}\right| \leq$ 
$A t^{\nu-1}, t \in\left(b_{n}^{i}, a_{n}^{i+1}\right]$, the integral $\int_{b_{n}^{i}}^{a_{n}^{i+1}} G(t) d t$ exists at least in the improper sense.

Lemma 1 Let $f$ be a Cantor type function on $C$ with $H$-dim $C=1-\nu$. Suppose that

1. $f_{H}^{\prime}(t)$ exists uniformly and is continuous for $t \in C$.

2. $\int_{0}^{t} u^{\nu-1} f_{H}^{\prime}(t-u) d u^{1-\nu}$ exists and is bounded for $t \in C$.

3. $f$ has self-similar property.

4. $f(0)=0$ and $\xi \leq \frac{1}{3}$.

Then there is a constant $A$ such that

$$
\frac{\left|\left(D^{-\nu} f\right)\left(b_{n}^{i_{t_{n}}}\right)-\left(D^{-\nu} f\right)\left(a_{n}^{i_{t_{n}}}\right)\right|}{\left(b_{n}^{i_{t}}\right)-\left(a_{n}^{i_{t}}\right)} \leq A
$$

where $a_{n}^{i_{t}} \leq t \leq b_{n}^{i_{t}}, t \in C$.

Proof. We may write $\left(D^{-\nu} f\right)\left(b_{n}^{i_{t_{n}}}\right)-\left(D^{-\nu} f\right)\left(a_{n}^{i_{t_{n}}}\right)$ as

$$
\begin{aligned}
\frac{1}{\Gamma(\nu)} \int_{0}^{a_{n}^{i_{n}}} u^{\nu-1}\left[f\left(b_{n}^{i_{t_{n}}}-u\right)-f\left(a_{n}^{i_{t_{n}}}-u\right)\right] d u & \\
& +\frac{1}{\Gamma(\nu)} \int_{a_{n}^{i_{t_{n}}}}^{b_{n}^{i_{n}}} u^{\nu-1} f\left(b_{n}^{i_{t_{n}}}-u\right) d u=I_{1}\left(t, h_{n}\right)+I_{2}\left(t, h_{n}\right) .
\end{aligned}
$$

Note that as $n$ increases the ratio $\frac{I_{2}\left(t, h_{n}\right)}{h_{n}}$ tends to zero. Therefore, there is a constant $A_{1}$ such that

$$
\left|\frac{I_{2}\left(t, h_{n}\right)}{h_{n}}\right| \leq A_{1}
$$

$\frac{I_{1}\left(t, h_{n}\right)}{h_{n}}$ may be expressed as

$$
\begin{gathered}
\frac{1}{h_{n}}\left[\int_{0}^{h_{n}}+\int_{b_{n}}^{b_{n}^{i_{t_{n}}}-b_{n}^{i_{t_{n}}-1}}+\int_{b_{n}^{i_{n}}-b_{n}^{i_{t_{n}}-1}}^{b_{n}^{i_{t_{n}}}-b_{n}^{i_{t_{n}}-1}+2 h}+\int_{b_{n}^{i_{n}}-b_{n}^{i_{t_{n}}-1}+2 h}^{b_{n}^{i_{n}}-b_{n}^{i_{t_{n}}-2}}+\cdots\right. \\
\left.\quad+\int_{b_{n}^{i_{t_{n}}}-b_{n}^{2}}^{b_{t_{n}}^{2}-b_{n}^{2}+2 h}+\int_{b_{n}^{i_{t_{n}}}-b_{n}^{2}+2 h}^{b_{t_{n}}^{2}-b_{n}^{1}} u^{\nu-1}\left[f\left(b_{n}^{i_{t_{n}}}-u\right)-f\left(a_{n}^{i_{t_{n}}}-u\right)\right] d u\right]
\end{gathered}
$$




$$
\begin{aligned}
& =\frac{1}{h_{n}}\left[\int_{0}^{h_{n}}+\int_{b_{n}^{i_{n}}-b_{n}^{i_{t_{n}}}-1}^{b_{i_{n}}^{i_{n}}-b_{n}^{i_{t_{n}}-1}+2 h}+\int_{b_{n}^{i_{n}}-b_{n}^{i_{t_{n}}-1}+2 h}^{b_{n}^{i_{t_{n}}}-b_{n}^{i_{t_{n}}-2}}+\cdots\right. \\
& \left.+\int_{b_{n}^{i_{n}}-b_{n}^{2}}^{b_{n}^{i_{t_{n}}}-b_{n}^{2}+2 h}+\int_{b_{n}^{i_{t_{n}}}-b_{n}^{2}+2 h}^{b_{n}^{i_{t_{n}}}-b_{n}^{1}} u^{\nu-1}\left[f\left(b_{n}^{i_{t_{n}}}-u\right)-f\left(a_{n}^{i_{t_{n}}}-u\right)\right] d u\right] .
\end{aligned}
$$

Using the identity in the proof of Theorem 3, we have

$$
\begin{aligned}
\sum_{j=1}^{i_{t_{n}}-2} \frac{1}{h_{n}} & \left(\int_{a_{n}^{i_{t_{n}}}-b_{n}^{i_{t_{n}}}-j}^{a_{n}^{i_{t_{n}}}-b_{n}^{i_{t_{n}}-j}+h_{n}}+\int_{a_{n}^{i_{t_{n}}}-b_{n}^{i_{t_{n}}-j}+h_{n}}^{a_{n}^{i_{n}}-b_{n}^{i_{t_{n}}-j}+2 h_{n}}\right) \\
& \times u^{\nu-1}\left[f\left(b_{n}^{i_{t_{n}}}-u\right)-f\left(a_{n}^{i_{t_{n}}}-u\right)\right] d u \\
= & \sum_{j=1}^{i_{t_{n}}-2}\left(a_{n}^{i_{t_{n}}}-b_{n}^{i_{t_{n}}-j}+\theta_{j} h_{n}\right)^{\nu-1}\left[f\left(b_{n}^{i_{t_{n}}-j}\right)-f\left(a_{n}^{i_{t_{n}}-j}\right)\right] \\
& +\sum_{j=1}^{i_{t_{n}}-2}\left[\left(a_{n}^{i_{t_{n}}}-b_{n}^{i_{t_{n}}-j}+\overline{1+\theta_{j}} h_{n}\right)^{\nu-1}-\left(a_{n}^{i_{t_{n}}}-b_{n}^{i_{t_{n}}-j}+\theta_{j} h_{n}\right)^{\nu-1}\right] \\
& \times \frac{1}{h_{n}} \int_{a_{n}^{i_{t_{n}}-j}}^{b_{n}^{i_{t_{n}}-j}}\left[f(u)-f\left(a_{n}^{i_{t_{n}}-j}\right)\right] d u .
\end{aligned}
$$

Now $\frac{I_{1}\left(t, h_{n}\right)}{h_{n}}$ may be displayed as

$$
\begin{aligned}
& \frac{1}{h_{n}} \int_{0}^{h_{n}} u^{\nu-1}\left[f\left(b_{n}^{i_{n}}-u\right)-f\left(a_{n}^{i_{t_{n}}}-u\right)\right] d u \\
& \quad+\sum_{j=1}^{i_{t_{n}}-2}\left(a_{n}^{i_{t_{n}}}-b_{n}^{i_{t_{n}}-j}+\theta_{j} h_{n}\right)^{\nu-1}\left[f\left(b_{n}^{i_{t_{n}}-j}\right)-f\left(a_{n}^{i_{t_{n}}-j}\right)\right] \\
& \quad+\left[\sum_{j=1}^{i_{t_{n}}-2}\left(a_{n}^{i_{t_{n}}}-b_{n}^{i_{t_{n}}-j}+\overline{1+\theta_{j}} h_{n}\right)^{\nu-1}-\left(a_{n}^{i_{t_{n}}}-b_{n}^{i_{t_{n}}}-j+\theta_{j} h_{n}\right)^{\nu-1}\right] \\
& \quad \times \frac{1}{h_{n}} \int_{a_{n}^{b_{t_{n}}-j}}^{b_{t_{n}}^{i_{t_{n}}}-j}\left[f(u)-f\left(a_{n}^{i_{t_{n}}-j}\right)\right] d u=I_{1.1}\left(t, h_{n}\right)+I_{1.2}\left(t, h_{n}\right)+I_{1.3}\left(t, h_{n}\right) .
\end{aligned}
$$

Clearly

$$
\left|I_{1.1}\left(t, h_{n}\right)\right| \leq \frac{C_{1}}{h_{n}} \int_{0}^{h_{n}} u^{\nu-1} h_{n}^{1-\nu} d u=\frac{C_{1}}{h_{n}} \frac{h_{n}^{\nu}}{\Gamma(\nu)} h_{n}^{1-\nu} \leq A_{2}
$$


where $A_{2}$ is a constant.

By condition (ii) there is a constant $C_{3}$ such that

$$
\left|\int_{0}^{t} u^{\nu-1} f_{H}^{\prime}(t-u) d u^{1-\nu}\right| \leq C_{3} .
$$

Let $K=I_{1.2}\left(t, h_{n}\right)-\int_{0}^{t} u^{\nu-1} f_{H}^{\prime}(t-u) d u^{1-\nu}$. To show that $K$ is bounded, we may write $K$ as

$$
\begin{aligned}
\sum_{j=1}^{i_{t_{n}}-2} \int_{a_{n}^{i_{t_{n}}-j}}^{b_{n}^{i_{t_{n}}-j}} & {\left[\left(a_{n}^{i_{t_{n}}}-b_{n}^{i_{t_{n}}-j}+\theta_{j} h_{n}\right)^{\nu-1}-(t-u)^{\nu-1}\right] f_{H}^{\prime}(u) d u^{1-\nu} } \\
& -\int_{0}^{t-a_{n}^{i_{t_{n}}}} u^{\nu-1} f_{H}^{\prime}(t-u) d u^{1-\nu}-\int_{t-b_{n}^{1}}^{t-a_{n}^{1}} u^{\nu-1} f_{H}^{\prime}(t-u) d u^{1-\nu} .
\end{aligned}
$$

Note that

$$
\begin{gathered}
\left(a_{n}^{i_{t_{n}}}-b_{n}^{i_{t_{n}}-j}+\theta_{j} h_{n}\right)^{\nu-1}-(t-u)^{\nu-1}=\frac{u_{j} h_{n}}{(\nu-1)\left(a_{n}^{i_{t_{n}}}-b_{n}^{i_{t_{n}}-j}+\lambda_{j} h_{n}\right)^{2-\nu}} \\
u \in\left[a_{n}^{i_{t_{n}}-j}-b_{n}^{i_{t_{n}}-j}\right], \quad 0 \leq u_{j}, \lambda_{j} \leq 2
\end{gathered}
$$

and

$$
\int_{a_{n}^{j}}^{b_{n}^{j}} u^{\nu-1} f_{H}^{\prime}(t-u) d u^{1-\nu} \leq D_{3} h_{n}^{\nu-1}, \quad D_{3} \text { constant. }
$$

Since $\xi \leq \frac{1}{3}$,

$$
\left(a_{n}^{i_{t_{n}}}-b_{n}^{i_{t_{n}}-j}+\theta_{j} h_{n}\right) \geq j h_{n} .
$$

We have

$$
\begin{aligned}
& \left|\sum_{j=1}^{i_{t_{n}}-2} \int_{a_{n}^{i_{t_{n}}-j}}^{b_{n}^{i_{t_{n}}-j}}\left[\left(a_{n}^{i_{t_{n}}}-b_{n}^{i_{t_{n}}-j}+\theta_{j} h_{n}\right)^{\nu-1}-(t-u)^{\nu-1}\right] f_{H}^{\prime}(u) d u\right| \\
& \quad \leq \sum_{j=1}^{i-2} \frac{2 D_{3} h_{n}^{1-\nu} h_{n}}{j^{2-\nu} h_{n}^{2-\nu}}=E_{3} .
\end{aligned}
$$

Therefore,

$$
|K| \leq E_{3}+C_{3}=A_{3}
$$

Final, let us estimate $I_{3}\left(t, h_{N}\right)$. As in the proof of Theorem $3, I_{3}\left(t, h_{n}\right)$ may be expressed as

$$
\sum_{j=1}^{i_{t_{n}}-2}\left[\left(a_{n}^{i_{t_{n}}}-b_{n}^{i_{t_{n}}-j}+\overline{1+\theta_{j}} h_{n}\right)^{\nu-1}-\left(a_{n}^{i_{t_{n}}}-b_{n}^{i_{t_{n}}-j}+\theta_{j} h_{n}\right)^{\nu-1}\right]
$$




$$
\begin{aligned}
& \times\left[\frac{1}{h_{n}} \int_{a_{n}^{i_{t_{n}}-j}}^{b_{n}^{i_{t_{n}}-j}}\left[f(u)-f\left(a_{n}^{i_{t_{n}}-j}\right)\right] d u-\beta \int_{a_{n}^{i_{t_{n}}-j}}^{b_{n}^{i_{t_{n}}-j}} f_{H}^{\prime}(u) d u^{1-\nu}\right] \\
& +\beta \sum_{j=1}^{i_{t_{n}}-2}\left[\left(a_{n}^{i_{t_{n}}}-b_{n}^{i_{t_{n}}-j}+\overline{1+\theta_{j}} h_{n}\right)^{\nu-1}-\left(a_{n}^{i_{t_{n}}}-b_{n}^{i_{t_{n}}}-j+\theta_{j} h_{n}\right)^{\nu-1}\right] \\
& \times \int_{a_{n}^{b_{n}-j}}^{b_{t_{n}}-j} f_{H}^{\prime}(u) d u^{1-\nu} .
\end{aligned}
$$

By the self-similarity of $f$, we have

$$
\begin{aligned}
\left|I_{3}\left(t, h_{n}\right)\right| \leq & F_{3} \sum_{j=1}^{i_{t_{n}}-2}\left[\left(a_{n}^{i_{t_{n}}}-b_{n}^{i_{t_{n}}-j}+\overline{1+\theta_{j}} h_{n}\right)^{\nu-1}\right. \\
& \left.-\left(a_{n}^{i_{t_{n}}}-b_{n}^{i_{t_{n}}}-j+\theta_{j} h_{n}\right)^{\nu-1}\right] \int_{a_{n}^{i_{n}}-j}^{b_{n}^{i_{t_{n}}-j}} f_{H}^{\prime}(u) d u^{1-\nu} .
\end{aligned}
$$

By an argument similar to that in the above paragraph

$$
\left|I_{3}\left(t, h_{n}\right)\right| \leq A_{4}
$$

combining (3), (6), (7), (8) we have

$$
\frac{\left|\left(D^{-\nu} f\right)\left(b_{n}^{i_{t_{n}}}\right)-\left(D^{-\nu} f\right)\left(a_{n}^{i_{t_{n}}}\right)\right|}{b_{n}^{i_{t_{u}}}-a_{n}^{i_{t_{u}}}} \leq A_{1}+A_{2}+A_{3}+A_{4}+C_{3}=A .
$$

We need one more concept to finish this article. Let $[a, b]$ be a closed interval, let $\left\{h_{n}\right\}_{n=1}^{\infty}$ be a sequence of positive numbers with $h_{n} \downarrow 0$. A division $D$ of $[a, b]$ is called an admissible division if $u=t-\alpha, v=t+\beta, \alpha=h_{n}$ or 0 , $\beta=h_{m}$ or 0 in the interval point pairs for all the $([u, v], t) \in D$ but associated point $t=b$.

Theorem 4 Let $f$ be a Cantor type function on $C$ with $H$-dim $C=1-\nu$, suppose that $f$ satisfies the conditions 1, 2, 3, 4 in Lemma 1. Then

$$
\left(D^{-\nu} f\right)(t)=\int_{0}^{t} \int_{0}^{u} v^{\nu-1} f_{H}^{\prime}(u-v) d v^{1-\nu} d u
$$

Proof. First let us show that for each interval $\left[b_{n}^{i}, a_{n}^{i+1}\right], i=1,2, \ldots, 2^{n}$, $n=1,2, \ldots$ and given $\varepsilon>0$, there is an admissible division $D$ such that

$$
\left|\left[\left(D^{-\nu} f\right)\left(a_{n}^{i+1}\right)-\left(D^{-\nu} f\right)\left(b_{n}^{i}\right)\right]-\int_{b_{n}}^{a_{n}^{i+1}} M(t) d t\right|
$$




$$
=\mid\left[(D) \sum\left[\left(D^{-\nu} f\right)(\beta)-\left(D^{-\nu} f\right)(\alpha)-\int_{\alpha}^{\beta} M(t) d t\right] \mid<\varepsilon\right.
$$

where $M(t)=\int_{0}^{t} v^{\nu-1} f_{H}^{\prime}(t-v) d v^{1-\nu}$.

Since $M(t)$ is a gauge integrable function in the interval $\left[b_{n}^{i}, a_{n}^{i+1}\right][6$, p. 112], for $\frac{\varepsilon}{2}>0$ there is a $\delta_{1}(t)$ such that

$$
\left|(D) \sum M(t)(\beta-\alpha)-\int_{b_{n}^{i}}^{a_{n}^{i+1}} M(t) d t\right|<\frac{\varepsilon}{2}
$$

For every $\delta_{1}$-fine division $D$.

By Theorem 3 for each $t \in\left(b_{n}^{i}, a_{n}^{i+1}\right]$ there is a $\delta_{2}(t)>0$ such that

$$
\begin{aligned}
\mid\left(D^{-\nu} f\right)(\beta)-\left(D^{-\nu} f\right)(\alpha) & -\int_{0}^{t} u^{1-\nu} f_{H}^{\prime}(t-u) d u^{1-\nu} \mid \\
& <\frac{\varepsilon}{3\left(a_{n}^{i+1}-b_{n}^{i}\right)}(\beta-\alpha)
\end{aligned}
$$

where $\alpha=t-h_{a}, \beta=t+h_{m}, 0<\beta-\alpha<\delta_{2}(t)$. Evidently there is a $\delta_{2}\left(b_{n}^{i}\right)$ such that

$$
\left|\int_{b_{n}^{i}}^{t} M(u) d u\right|<\frac{\varepsilon}{6}
$$

and

$$
\left|\left(D^{-\nu} f\right)(t)-\left(D^{-\nu} f\right)\left(b_{n}^{i}\right)\right|<\frac{\varepsilon}{6}
$$

where $0<t-b_{n}^{i}<\delta_{2}\left(b_{n}^{i}\right)$.

Let $\delta(t)=\min \left(\delta_{1}(t), \delta_{2}(t)\right)$. It is a routine argument to check there is a $\delta$-fine admissible division $D$ of $\left[b_{n}^{i}, a_{n}^{i+1}\right]$. We may write

$$
\left[\left(D^{-\nu} f\right)\left(a_{n}^{i+1}\right)-\left(D^{-\nu} f\right)\left(b_{n}^{i}\right)-\int_{b_{n}^{i}}^{a_{n}^{i+1}} M(t) d t\right.
$$

as

$$
\begin{aligned}
(D) \sum & {\left[\left(D^{-\nu} f\right)(\beta)-\left(D^{-\nu} f\right)(\alpha)\right]-(D) \sum \int_{\alpha}^{\beta} M(t) d t } \\
= & (D) \sum^{\prime}\left[\left(D^{-\nu} f\right)(\beta)-\left(D^{-\nu} f\right)(\alpha)\right]+\left(D^{-\nu} f\right)\left(\beta_{n}^{i}\right)-\left(D^{-\nu} f\right)\left(b_{n}^{i}\right) \\
& -(D) \sum^{\prime} \int_{\alpha}^{\beta} M(t) d t-\int_{b_{n}^{i}}^{\beta_{n}^{i}} M(t) d t
\end{aligned}
$$


where $\left(\left[b_{n}^{i}, \beta_{n}^{i}\right], b_{n}^{i}\right) \in D$ and $(D) \sum^{\prime}=(D) \sum \backslash\left(\left[b_{n}^{i}, \beta_{n}^{i}\right], b_{n}^{i}\right)$. Then

$$
\begin{aligned}
(D) \sum & {\left[\left(D^{-\nu} f\right)(\beta)-\left(D^{-\nu} f\right)(\alpha)\right]-(D) \sum \int_{\alpha}^{\beta} M(t) d t } \\
= & (D) \sum^{\prime}\left[\left(D^{-\nu} f\right)(\beta)-\left(D^{-\nu} f\right)(\alpha)-\int_{0}^{t} u^{\nu-1} f_{H}^{\prime}(t-u) d u^{\nu-1}(\beta-\alpha)\right] \\
& +(D) \sum^{\prime}\left[\int_{0}^{t} u^{\nu-1} f_{H}^{\prime}(t-u) d u^{\nu-1}(\beta-\alpha)-\int_{\alpha}^{\beta} M(t) d t\right] \\
& +\left(D^{-\nu} f\right)\left(\beta_{n}^{i}\right)-\left(D^{-\nu} f\right)\left(b_{n}^{i}\right)-\int_{b_{n}^{i}}^{\beta_{n}^{i}} M(t) d t \\
= & I_{1}+I_{2}+I_{3} .
\end{aligned}
$$

Compute $I_{1}$ by (2)

$$
\left|I_{1}\right| \leq(D) \sum^{\prime} \frac{\varepsilon}{b\left(a_{n}^{i_{n}}-b_{n}^{i}\right)}(\beta-\alpha)<\frac{\varepsilon}{3} .
$$

By Henstock's lemma, we have $\left|I_{2}\right|<\frac{\varepsilon}{2}$. Combining (3), (4), gives $\left|I_{2}\right|<\frac{\varepsilon}{3}$ as required.

By changing the index we may write $\left\{\left[b_{n}^{i}, a_{n}^{i+1}\right]_{i=1}^{2^{n}-1}\right\}_{n=1}^{\infty}$ as $\left\{\left[\alpha_{m}, \beta_{m}\right]_{m=1}^{\infty}\right\}$. For each $\left[\alpha_{m}, \beta_{m}\right]$ there is a $\delta_{m}(t)$ defined on $\left\{\left[\alpha_{m}, \beta_{m}\right]\right.$ such that

$$
\left|(D) \sum M(t)(\beta-\alpha)-\int_{\alpha_{m}}^{\beta_{m}} M(t) d t\right|<\frac{\varepsilon}{2^{m+1}}, \quad m=1,2, \ldots
$$

for every $\delta_{m}$-fine division $D$.

Let $A$ be the constant in Lemma 1 and let $\delta_{0}(t)=\eta$ be small enough. Let

$$
\delta(t)=\left\{\begin{array}{l}
\delta_{0}, t \in C \\
\delta_{m}(t), t \in\left[\alpha_{m}, \beta_{m}\right]
\end{array}\right.
$$

By a covering lemma $[2$, p. 4], there is a net $\delta$-fine division $D$ of $[0, T]$. The $\left(D^{-\nu} f\right)(t)-\int_{0}^{t} M(u) d u$ may be displayed as

$$
\begin{aligned}
(D) & \sum\left[\left(D^{-\nu} f\right)(\beta)-\left(D^{-\nu} f\right)(\beta)-\int_{\alpha}^{\beta} M(u) d u\right] \\
& =\left[\left(D_{1}\right) \sum+\left(D_{2}\right) \sum\right]\left[\left(D^{-\nu} f\right)(\beta)-\left(D^{-\nu} f\right)(\alpha)-\int_{\alpha}^{\beta} M(u) d u\right]
\end{aligned}
$$




$$
=J_{1}+J_{2}
$$

where $D_{1}$ denotes the collection of elements with associated point $t \in C$ and $D_{2}$ the collection of elements with associated point $t \in[0, T] \backslash C$. Clearly

$$
\left|J_{1}\right| \leq\left(D_{1}\right) \sum A(\beta-\alpha)+\left(D_{1}\right) \sum C_{3}(\beta-\alpha) \leq\left(A+C_{3}\right)(2 \xi)^{n},
$$

where $C_{3}$ denotes the upper bounded of $M(t)$.

$$
\left|J_{2}\right| \leq\left(D_{2}\right) \sum \frac{\varepsilon}{2^{m}} \leq \frac{\varepsilon}{2}
$$

Take $\eta$ small enough such that

$$
\left(A+C_{3}\right)(2 \xi)^{n}<\frac{\varepsilon}{2} .
$$

Combining (13), (14)

$$
\left|\left(D^{-\nu} f\right)(t)-\int_{0}^{t} M(u) d u\right|<\varepsilon .
$$

Since $\varepsilon$ arbitrary, we have

$$
\left(D^{-\nu} f\right)(t)=\int_{0}^{t} \int_{0}^{u} v^{\nu-1} f_{H}^{\prime}(u-v) d v^{1-\nu} d u
$$

Corollary 1 Let $f$ be the function in Theorem 4. Then

$$
\left(D^{-\nu} f\right)(t)=\int_{0}^{t} u^{1-\nu} J_{\nu}\left(v f_{H}^{\prime}(u-v) d u .\right.
$$

Proof. Using the identity $[2$, p. 7]

$$
\int_{0}^{u} v^{\nu-1} f_{H}^{\prime}(u-v) d v^{1-\nu}=u^{1-\nu} J_{\nu}\left(v f_{H}^{\prime}(u-v)\right) .
$$

Remark 3 Let $f \in C^{1}, f(0)=0$, It is easy to show

$$
\left(D^{-\nu} f\right)(t)=\int_{0}^{t} \int_{0}^{u} v^{\nu-1} f^{\prime}(u-v) d v d u .
$$

This identity is similar to the above identity, but the function $f$ is smooth. Note that in our discussion the function $f$ is singular.

Acknowledgments. The author wishes to thank Prof. Peter S. Bullen and the referee for their useful suggestions which improved the paper substantially. 


\section{References}

[1] SS. Fu, ג-power integrals on the Cantor type sets, Proc. Amer. Math. Soc., 123 (1995) 2731-2737.

[2] SS. Fu, S-dimensional Hausdorff integral and its physical interpretation, Real Analysis Exchange 21 (1995-96), 308-316.

[3] G. H. Hardy and J. E. Littlewood, Some properties of fractional integrals. I, Math Zeitschrift, 27 (1928), 565-606, (particularly Theorems 12-20 on pp. 584-592).

[4] S. Kempfle, Note on R. R. Nigmatullin identity between a Cantor Dust convolution and temporal fractional integral, preprint, 1994.

[5] B. B. Mandelbrot, The fractal geometry of nature, San Francisco, W. H. Freonan \& Co., 1982.

[6] E. J. McShane, Unified integration, Academic Press. Inc, 1982.

[7] R. R. Nigmatullin, Fractional integral and its physical interpretation, Theor. Math. Phys., 90 (1992), 243-251.

[8] R. S. Rutman, On the paper by R. R. Nigmatullin. Fractional integral and its physical interpretation, Theor. Math. Phys., 100 (1994), 476-478.

[9] B. S. Thomson, Derivates of interval functions, Mem. Amer. Math. Soc., 93 (1991).

[10] C. Tricot, Dérivées fractionaires et dimension Fractale, Ecole Polytechnique de Montréal, 1994. 